\begin{tabular}{l} 
PHILOSOPHICAL \\
TRANSACTIONS \\
$\begin{array}{l}\text { THE ROYAL } \\
\text { SOCIETY }\end{array}$ \\
\hline
\end{tabular}

\title{
Measure recognition problem
}

\author{
By Mirna Džamonja* \\ School of Mathematics, University of East Anglia, Norwich NR4 7TJ, UK
}

This is a paper in mathematics, specifically in set theory. On the example of the measure recognition problem (MRP), the paper highlights the phenomenon of the utility of a multidisciplinary mathematical approach to a single mathematical problem, in particular, the value of a set-theoretic analysis. MRP asks if for a given Boolean algebra, $\mathfrak{B}$, and a property, $\Phi$, of measures, one can recognize by purely combinatorial means if $\mathfrak{B}$ supports a strictly positive measure with property $\Phi$. The most famous instance of this problem is MRP (countable additivity), and in the first part of the paper, we survey the known results on this and some other problems. We show how these results naturally lead to asking about two other specific instances of the problem MRP, namely MRP (non-atomic) and MRP (separable). Then, we show how our recent work gives an easy solution to the former of these problems and some partial information about the latter. The long-term goal of this line of research is to obtain a structure theory of Boolean algebras that support a finitely additive strictly positive measure, along the lines of Maharam theorem, which gives such a structure theorem for measure algebras.

Keywords: set theory; Boolean algebras; measure; combinatorial characterization

\section{Introduction}

Having announced that this is a paper in set theory, let us commence by some remarks on the nature of that subject. Set theory is a part of mathematical logic and, indeed, it has a dual role of giving logical foundations to mathematics and of being a part of mathematics itself. In many people's minds, these two roles are rather distinct. In fact, much of the development of set theory in the twentieth century ran on two distinct tracks. On the one hand, much effort was spent to develop systems of set-theoretic axioms from which one could logically develop the known mathematics. Hilbert's programme made it a priority to have such a system, and it had widely been believed after the set theory developed by Cantor (1874), in the late nineteenth century, that one should be able to have an axiomatic system for mathematics by using the notion of a set as given. The present understanding of this is rather different, mostly due to the work of Gödel (1931) in his famous Incompleteness Theorems. Firstly, he showed that for every consistent recursive system of axioms, which includes the Peano Arithmetic, there is a statement formalizable in the theory itself, which is independent (neither provable nor unprovable) in the theory, and secondly that such systems of axioms also cannot prove their own consistency. On the other hand, much

*h020@uea.ac.uk

One contribution of 23 to a Triennial Issue 'Mathematics and physics'. 
understanding was developed about the mathematical, rather than the logical, consequences of Cantor's work. This includes the idea of infinite sets of various sizes and the understanding of what this means in terms of familiar objects, such as the sets of real numbers. This paper will mainly concentrate on that mathematical side of set theory, but in fact, the point is that the two sides of set theory are indivisible, as we wish to demonstrate by this paper.

Most (but not all) of the modern mathematics takes the axiom scheme known as Zermelo-Fraenkel axioms with Choice (ZFC) as a basis. It has been the case basically since the 1920s and there are many good reasons for this decision. Some may then view it as a serious setback that it became known since Cohen (1963) that, as we know from Gödel (1931), there are not only some statements of mathematics that are independent of ZFC, but also some ordinary statements, notably the Continuum Hypothesis ('every infinite subset of the set $\mathbb{R}$ of real numbers is in a bijective correspondence either with the set $\mathbb{N}$ of natural numbers or the set $\mathbb{R}$ itself'), that are independent. This was established by showing that one cannot calculate the size $|\mathbb{R}|=|\mathcal{P}(\mathbb{N})|$ just by using the axioms of ZFC in terms of the Cantor's $\boldsymbol{\aleph}$-hierarchy of infinite cardinals: $\left|\boldsymbol{\aleph}_{0}\right|=|\mathbb{N}|$, the next infinite cardinal $\boldsymbol{\aleph}_{1}, \boldsymbol{\aleph}_{2}, \ldots$ the limit $\boldsymbol{\aleph}_{\omega}$, the next $\boldsymbol{\aleph}_{\omega+1}, \ldots$. Moreover, it is consistent with these axioms (assuming they themselves are consistent) that the value of $\left|\mathcal{P}\left(\boldsymbol{\aleph}_{0}\right)\right|$ is as large as desired, i.e. no upper bound can be found just by arguing in ZFC. Indeed, Cohen's result entirely changed the subject of set theory, not only owing to its logical significance but also because it introduced a method for proving that various statements were independent of ZFC. This is the method of forcing. Many interesting results have been obtained by applying this method. While this is exciting, it also created the feeling in the general mathematical community that set theorists are mostly concerned about things that cannot be done, rather than the ones that can be. It did not help that the forcing machinery had developed to an incredible extent and even being able to read and verify some of the proofs takes an enormous effort. There was definitely a period in which the set theory was considered far removed from the interests of the main stream mathematics.

I am very fortunate to belong to the generation of mathematicians who have now seen the pendulum swing the other way. In the recent years, there have been a number of purely mathematical results that have been obtained as a consequence of a fine set-theoretic analysis of the problem. The final result often does not mention any set-theoretic assumptions additional to ZFC, which is assumed throughout, yet the proof relies deeply on an understanding of the settheoretic limits of ZFC and its possible universes. As an example, let us state a celebrated theorem of Shelah (1994):

Theorem 1.1 (Shelah). If $\left|\mathcal{P}\left(\boldsymbol{\aleph}_{n}\right)\right|<\boldsymbol{\aleph}_{\omega}$ for all $n<\omega$, then $\left|\mathcal{P}\left(\boldsymbol{\aleph}_{\omega}\right)\right|<\boldsymbol{\aleph}_{\omega_{4}}$.

To appreciate the impact of the above theorem, contrast it with what we have already said, it is not possible to bound $\left|\mathcal{P}\left(\boldsymbol{\aleph}_{n}\right)\right|$ by arguing in ZFC. However, if we are in the situation to know that this value for every $n$ is less than $\boldsymbol{\aleph}_{\omega}$, then we can put a definite bound on the size of $\left|\mathcal{P}\left(\boldsymbol{\aleph}_{\omega}\right)\right|$. This theorem as stated can be understood without any prior knowledge of modern set theory. Yet, this statement is a culmination of at least 20 years of concentrated effort by many set theorists, involving techniques such as large cardinals, iterated forcing, elementary embeddings and culminating by the seminal work of Shelah 
(started in the 1980s and presented as a whole in Shelah (1994)) in which he invented the whole theory (the theory of possible cofinalities, 'pcf') to finally prove the theorem. This part of Shelah's work is the one that was cited when he was awarded the prestigious Bolyai Prize.

In this paper, we shall concentrate on the appearance of this phenomenon of the symbiosis between the axiomatic and the mathematical in the context of measure theory. We shall describe the problem of characterizing Boolean algebras that carry a measure, and point out the most well-known instance of this problem. This is of course the von Neumann (1981) problem, recently solved in the negative by Talagrand (submitted). We shall then consider some other instances of this problem and show some recent solutions that have been obtained in our joint work by Džamonja \& Plebanek (submitted).

\section{Description of the problem}

In order to make the paper accessible, we shall commence with a quick review of the basic notions we use. A list of notational conventions can be found at the end of this paper.

A Boolean algebra, $\mathfrak{B}$, is a structure consisting of a set with at least two distinct elements, $0_{\mathfrak{B}}$ and $1_{\mathfrak{B}}$, two binary operations, $\wedge$ and $\vee$, and a unary operation, -, which obeys certain rules known as the laws of Boolean algebras. A typical example of a Boolean algebra is a family of subsets of a given set $A$, where $0_{\mathfrak{B}}=0,1_{\mathfrak{B}}=A$, and the operations, $\wedge, \vee$ and - , are interpreted as $\cap, \cup$ and the complement, $c$, respectively. It follows from Stone Representation Theorem (Stone 1936) that every Boolean algebra is isomorphic as a structure to some $\mathfrak{B} \subseteq \mathcal{P}(A)$ for some $A$, so we shall only work with such algebras. The basic laws of Boolean algebras are then interpreted as the familiar commutativity, associativity and distributivity laws between $U$ and $\cap$, and we also have that for any $a \in \mathfrak{B},\left(a^{c}\right)^{c}=a$. These operations induce the familiar subset relation, $\subseteq$, which acts as a relation of partial ordering on $\mathfrak{B}$. Two elements $a, b$ of $\mathfrak{B}$ are said to be disjoint if $a \cap b=0$.

Boolean algebras, $\mathfrak{B}$, may also have properties additional to the ones given by the basic laws. For example, we can consider the antichains, which are subsets of $\mathfrak{B}$ consisting of pairwise disjoint elements. The condition which guarantees that all the antichains in $\mathfrak{B}$ are countable is called the countable (anti) chain condition and abbreviated as ccc. An example of a Boolean algebra that satisfies this law is the family $\mathcal{M}$ of the equivalence classes (mod measure 0 ) of all Lebesgue measurable subsets of the unit interval $[0,1]$. This is easily seen and is due to the additivity properties of the Lebesgue measure $\lambda$. It is known (see Fremlin 1989) that one can choose the representatives, $E^{\bullet}$, of the equivalence classes of measurable sets $E$ so that $\emptyset^{\bullet}=\emptyset$ and the representative of $E^{\bullet} \cup(\cap) F^{\bullet}$ is the union (intersection) of the corresponding representatives, for all $E^{\bullet}, F^{\bullet}$. (For this reason, we omit ${ }^{-}$in our notation.) Another property of $\mathcal{M}$ is that for every sequence $\left\langle a_{n}: n<\omega\right\rangle$ in $\mathcal{M}$ the union $\cup_{n<\omega} a_{n}$ is an element of $\mathcal{M}$, and it is the least upper bound of $\left\langle a_{n}: n<\omega\right\rangle$ with respect to $\subseteq$. Boolean algebras with this property are said to be $\sigma$-complete. In fact, the analogous completeness property remains true for sequences indexed by any other ordinal but $\omega$, which can be proved by using the ccc property along with the $\sigma$-completeness. This property is called 
completeness, and since in this paper, we never deal with algebras that are $\sigma$-complete without being complete, we shall simplify the notation and refer to both concepts by the word 'complete'.

Note that in the above example, $\lambda$ induces a function on $\mathcal{M}$, which is again called $\lambda$, and this function satisfies the following, for all $a, b, a_{n} \in \mathcal{M}$ :

(i) (strict positivity) $a \neq \emptyset \Rightarrow \lambda(a)>0, \lambda(\emptyset)=0$,

(ii ${ }^{-}$) (total finiteness) $\lambda\left(1_{\mathcal{M}}\right)<\infty$,

(iii) (additivity) if $a, b$ are disjoint then $\lambda(a \cup b)=\lambda(a)+\lambda(b)$, moreover

$\left(\right.$ iii $\left.^{+}\right)$(countable additivity) if $\left\langle a_{n}: n<\omega\right\rangle$ are pairwise disjoint, then $\lambda\left(\cup_{n<\omega} a_{n}\right)=\sum_{n<\omega} \lambda\left(a_{n}\right)$.

A complete Boolean algebra, $\mathfrak{B}$, which supports a functional $\lambda$ satisfying properties (i), (ii ${ }^{-}$) and $\left(\right.$iii $^{+}$) above is called a measure algebra and $\lambda$ is called a strictly positive (s.p.) countably additive measure. Measures which only satisfy properties (i), (ii ${ }^{-}$) and (iii) are called s.p. finitely additive measures. Since it is clear that by multiplying by a constant, we can obtain from $\lambda$ another countably additive measure $\mu$ which satisfies $\mu\left(1_{\mathcal{M}}\right)=1$ (such measures are called probabilities), we can replace the requirement (ii ${ }^{-}$) in the definition of a measure algebra by the one requiring the relevant measure to be a probability. In the sequel, the word 'measure' will refer to finitely additive measures on algebras which are not necessarily complete. The classical theory of such measures may be found in Bhaskara Rao \& Bhaskara Rao (1983).

The general problem we shall discuss in this paper is the following:

Measure Recognition Problem $\operatorname{MRP}(\Phi)$. Given a Boolean algebra, $\mathfrak{B}$, and a property, $\Phi$, of measures, how can we recognize by purely combinatorial means if on $\mathfrak{B}$ one can define a strictly positive measure with property $\Phi$ ?

\section{Comments on the problem and known results}

The most well-known instance of the MRP was asked by von Neumann in 1937 (see von Neumann 1981). He was interested in recognizing the measure algebras (so the additional property $\Phi$ in the problem description is the property of countable additivity and we are dealing with MRP (countably additive)). In addition to the completeness and the ccc property, von Neumann isolated another property which is always present in measure algebras, called weak distributivity, and asked if these three properties together were sufficient for a Boolean algebra to be a measure algebra. This famous problem was answered only very recently by Talagrand (submitted), and the answer is negative. In fact, the main result of Talagrand (submitted) answered, also negatively, the related well-known Control Measure Problem, which asks if the existence of a so-called continuous submeasure on a $\sigma$-complete Boolean algebra implies the existence of a measure. The step from this solution to the solution of von Neumann's problem then followed by known work, as explained in Talagrand's paper. A (strictly positive) continuous submeasure is a finite non-negative function, $\mu$, on a Boolean algebra, $\mathfrak{B}$, vanishing only at $0_{\mathfrak{B}}$, having the 'submeasure' property that $\mu(a \cup b) \leq \mu(a)+\mu(b)$ for all $a, b \in \mathfrak{B}$, and the 'continuity' property that for any sequence $\left\langle a_{n}: n<\omega\right\rangle$ of elements of $\mathfrak{B}$ satisfying $a_{n+1} \subseteq a_{n}$ for all $n$ and $\cap_{n<\omega} a_{n}=0$, 
we have $\lim _{n} \mu\left(a_{n}\right)=0$. The result of Talagrand (submitted) is probably the most exciting recent result to come out of a whole variety of mathematical subjects, particularly measure theory and set theory.

The notion of a continuous submeasure comes from the work of Maharam (1947), who observed that a necessary condition for a Boolean algebra to be a measure algebra is to be metrizable, and showed how one can define a continuous submeasure from the appropriate metric. Following this work, an important question became to recognize Boolean algebras that support continuous submeasures, the so-called Maharam's algebras. Recently, it was proved in Balcar et al. (2005) and Veličković (2005) that under a certain set-theoretic axiom known as the P-ideal dichotomy, any ccc weakly distributive complete Boolean algebra is a Maharam algebra. Using their work as a starting point, Todorčević (2004) obtained the following interesting characterization:

Theorem 3.1 (Todorčević). A complete Boolean algebra carries a strictly positive continuous submeasure if and only if it is weakly distributive and satisfies the $\sigma$-finite chain condition.

The chain condition here means that the algebra can be written as a countable union of subsets each of which only has finite antichains. Todorčević's result is clearly a theorem of ZFC, but it was obtained as a consequence of a set-theoretic analysis of the situation under the P-ideal dichotomy, and the methods introduced in Maharam (1947). Likewise, Talagrand's result is purely a theorem of ZFC, but even the formulation by Maharam of the Control Measure Problem was a consequence of her analysis of the behaviour of the von Neumann's problem under the set-theoretic assumption of the existence of a Souslin tree. Both of these results illustrate the point that this paper makes, about the close connection between a set-theoretic analysis of a problem and its solution in ZFC, as well as the long-term view that one has had to take in understanding various specific instances of this connection.

Next, let us take a look at another special case of MRP, namely $\operatorname{MRP}(\emptyset)$, the situation when we do not require any special property $\Phi$. In this case, there is a combinatorial criterion due to Kelley (1959). It involves the notion of the intersection number of a family $\mathcal{F}$ of sets, which is defined to be the supremum of all $\alpha$, such that for every finite subsequence $\bar{a}$ of elements of $\mathcal{F}$ (with possible repetitions of elements), there is a subsequence $\bar{b}$ of length $\lg (\bar{b})$ at least $\alpha \lg (\bar{a})$, such that the intersection of all elements of $\bar{b}$ is non-empty. Kelley's criterion is then:

Theorem 3.2 (Kelley). A Boolean algebra $\mathfrak{B}$ carries a strictly positive (finitely additive) measure if and only if $\mathfrak{B} \backslash\{\emptyset\}$ can be written as a countable union of families each of which has positive intersection number.

One may wonder how strong the condition in the Kelley's criterion is. It clearly implies the ccc, but Gaifman (1964) proved that there are ccc algebras which do not satisfy Kelley's criterion. It is also known that under the set-theoretic statement known as Martin's Axiom (MA) and the negation of the continuum hypothesis $(\mathrm{CH})$, all ccc Boolean algebras of size $<2^{\mathrm{\aleph}_{0}}$ do satisfy Kelley's condition and in fact some stronger conditions (see Fremlin 1984). This together with the example of the P-ideal dichotomy results quoted above demonstrates that there are some mathematical axioms, which make it easier for a Boolean algebra to have certain measure-theoretic properties. We shall see another example of this 
behaviour in $\S 4$. Once the notion of the intersection number is known, the proof of Kelley's theorem follows rather readily by the well-known facts from functional analysis. It seems to be a tendency in this subject that positive theorems once formulated properly, have proofs which are much less involved than the proofs of the negative ones. We shall illustrate this in theorem 4.1, where we shall give a proof of one such positive theorem, while we may note that most of the negative statements mentioned here (e.g. the construction from Talagrand (submitted)) have proofs that are very much out of the scope of this paper.

Special cases of property $\Phi$ which are particularly interesting are the notions of non-atomicity and separability. To motivate the definitions, we shall go back to the case of countably additive measures. An example of such a measure is the familiar Lebesgue measure $\lambda$ on the unit interval. This measure naturally leads to a measure on the Tychonoff product $[0,1]^{\kappa}$ for any cardinal $\kappa$, denoted by $\lambda_{\kappa}$. A remarkable theorem of Maharam (1942) shows that the structure of measure algebras (so supporting a countably additive measure) is totally determined by these examples. Namely, any measure algebra can be decomposed into a countable union of subsets, each of which is either an atom, or a Boolean algebra isomorphic to the measure algebra $\mathfrak{B}_{\kappa}$ of some $[0,1]^{\kappa}$ under $\lambda_{\kappa}$, where all $\kappa$ are infinite cardinals. Here, we use the notion of an atom of a Boolean algebra $\mathfrak{B}$, which is an element $a \neq 0$ such that only $b \subseteq a$ in $\mathfrak{B}$ are 0 and $a$. Such a structure theorem does not exist for Boolean algebras that simply support a strictly positive finitely additive measure. Maharam (1981) asks exactly for such a theorem.

Taking a long-term goal to obtain such a structure theory for finitely additive measures, we may ask ourselves what the first step would be. In the case of countably additive measures such a step was to determine the relevant building blocks, namely the algebras $\mathcal{B}_{\kappa}$. Each of these blocks has two important properties. The first one is that the measure is non-atomic, which means that for every $\varepsilon>0$, there is a finite partition of the algebra into elements of measure $<\varepsilon$. For this reason, we wish to have a combinatorial criterion for recognizing when a Boolean algebra has a finitely additive measure which is non-atomic, which explains why we believe the case of MRP (non-atomic) is an important special case. In our recent work by Džamonja \& Plebanek (submitted), we obtained a simple solution to this problem, which will be presented in $\$ 4$.

Another property of measure algebras, $\mathcal{B}_{\kappa}$, for $\kappa \geq \boldsymbol{\kappa}_{0}$ is that they can be understood as metric spaces of density $\kappa$. Namely one introduces a metric $d_{\kappa}$ derived from $\lambda_{\kappa}$ by declaring $d_{\kappa}(a, b)=\lambda_{\kappa}(a \Delta b)$, and this metric has the required property. By Maharam's theorem mentioned earlier, every measure algebra, $\mathfrak{B}$, has a (unique) decomposition involving algebras $\mathfrak{B}_{\kappa}$ for some $\kappa$, and the supremum of all $\kappa$ involved in this composition is called Maharam's dimension or type of $\mathfrak{B}$. Hence, it would be of interest to have a similar notion for Boolean algebras which simply support a finitely additive strictly positive measure. The notion of type can be defined similarly to the above because a finitely additive strictly positive measure on $\mathfrak{B}$ will already induce a metric on $\mathfrak{B}$, and we can make a reasonable definition of the type of $\mathfrak{B}$ to be the supremum of all densities of metric spaces obtained from $\mathfrak{B}$ by using all the possible strictly positive measures on $\mathfrak{B}$ (of course the actual calculation of the type in this case, in the absence of a representation theorem, may be very difficult). In the same vein, for a fixed measure $\mu$ on $\mathfrak{B}$, we may consider the density of the induced metric space. In particular, we say that $\mu$ is separable if the induced metric space is separable 
in the topological sense (i.e. it has a countable dense subset). Recognizing Boolean algebras that have such a strictly positive measure translates in the context of MRP into MRP (separable). Clearly, a similar notion can be defined for any fixed possible densities of a metric space, but for the moment, we still do not know how to solve MRP (separable). We shall show some partial solutions in $\$ 4$.

\section{Some recent results}

In this section, we shall take for granted all notions defined in previous sections. Here, we concentrate on MRP with two specific values of $\Phi$ in mind, MRP (nonatomic) and MRP (separable). All otherwise unattributed theorems are from Džamonja \& Plebanek (submitted). In particular, this work solves the problem MRP (non-atomic) mentioned earlier, using a rather simple argument. We present the argument here:

Theorem 4.1. A Boolean algebra $\mathfrak{B}$ carries a strictly positive non-atomic measure if and only if there is a decomposition $\mathfrak{B} \backslash\{0\}=\cup_{n<\omega} \mathfrak{B}_{n}$, where for each $n$ we have

(i) $\mathfrak{B}_{n} \subseteq \mathfrak{B}_{n+1}$,

(ii) $\operatorname{int}\left(\mathfrak{B}_{n}\right) \geq 2^{-n}$, and

(iii) if $a \in \mathfrak{B}_{n}$ then there are disjoint $b, c \in \mathfrak{B}_{n+1}$ with $b \cup c \subseteq a$.

The forward direction of this theorem is easy modulo known facts. For example, one can use the well-known Stone duality theory between Boolean algebras and compact zero-dimensional topological spaces (Stone 1936) and its application to measure algebras to transfer the problem into the setting of Radon measures on compact spaces. The conclusion then follows by another well-known theorem, Maharam (1942). Details are not of interest here. However, we shall sketch the proof of the backward direction of the theorem, assuming the following lemma which appears as part of Kelley's (1959) proof and is taken in this form from Fremlin (2002), Proposition 391 I. The notation $\operatorname{int}(A)$ stands for the intersection number of the family $A$.

Lemma 4.2 (Kelley). Let $\mathfrak{A}$ be a Boolean algebra and $A \subseteq \mathfrak{A} \backslash\{0\}$ non-empty. Then

$$
\operatorname{int}(A)=\max _{\nu} \inf _{a \in A} \nu(A)
$$

where the maximum is taken over all probability (finitely additive) measures on $\mathfrak{A}$.

Proof. If there is a decomposition of $\mathfrak{B}$ satisfying (i)-(iii), then by lemma 4.2 for each $n$, we can define a probability measure $\mu_{n}$ on $\mathfrak{B}$ such that for all $b \in \mathfrak{B}_{n}$ we have $\mu_{n}(b) \geq 2^{-n}$. We let $\mu$ be any cluster point of the sequence be $\left\langle\mu_{n}: n<\omega\right\rangle$. It is easily seen that is a probability measure on $\mathfrak{B}$. Let us show that $\mu$ is strictly positive. By induction on $n$, it easily follows that for all $a \in \mathcal{B}_{n}$ and $k \geq n$, there are $2^{k-n}$ pairwise disjoint sets in $\mathcal{B}_{k}$ contained in $a$. Hence, for such $n, k$ we have $\mu_{k}(a) \geq 2^{k-n} \cdot 2^{-k}=2^{-n}$. Consequently, $\mu(a) \geq 2^{-n}>0$.

Suppose now that $\varepsilon>0$ and $a \in \mathfrak{B} \backslash\{0\}$ are given. Let $n$ and $k \geq n$ be large enough so that $a \in \mathcal{B}_{n}$ and $2^{k-n}<\varepsilon$, so $\varepsilon \cdot 2^{k-n}>1$. Let $b_{0}, \ldots, b_{2^{k-n}-1}$ be disjoint elements of $\mathcal{B}_{k}$ contained in $a$, which exist as shown in the previous paragraph. Then $\mu\left(b_{i}\right)<\varepsilon$ for at least one $i<2^{k-n}-1$, by the choice of $k$. This shows that every non-zero element of $\mathfrak{B}$ has a subset of arbitrarily small positive measure. 
An essential difference between this criterion and other combinatorial criteria we mentioned above (Maharam, Kelley and Todorčević), is that the decomposition of the Boolean algebra involves an interaction between the countably many pieces involved. Next, one may wonder how strong this criterion is. Obviously, a necessary condition on the Boolean algebra to support a nonatomic strictly positive measure is first of all that it supports any strictly positive measure, so Kelley's criterion, and secondly that the algebra itself is atomless, which means that it does not have any atoms in the sense defined in $\S 3$. Džamonja \& Plebanek (submitted) give an example showing that, in general, these conditions are not sufficient to show that the algebra supports a non-atomic strictly positive measure. As it is known that under the axiom MA various properties of Boolean algebras of size smaller than $2^{\aleph_{0}}$ tend to be equivalent, the following theorem is perhaps not surprising:

Theorem 4.3. Assume MA. Then for atomless Boolean algebras $\mathcal{B}$ of size $<2^{\mathrm{x}_{0}}$, the following are equivalent:

(i) $\mathcal{B}$ is $c c c$, and

(ii) $\mathcal{B}$ satisfies the condition from theorem 4.1.

What may be surprising is the way that the theorem is proved. We give an informal sketch, for which we need several notions. This will also be a good opportunity to introduce an auxiliary property of Boolean algebras which was central in Džamonja \& Plebanek (submitted), the so-called approximability.

A forcing notion is a partially ordered set $\boldsymbol{P}$ with the least element ${ }^{1}$. Two elements of $\boldsymbol{P}$ are incompatible if there is no element of $\boldsymbol{P}$ that is larger than both of them. We say that $\boldsymbol{P}$ is ccc if there is no uncountable family of pairwise incompatible elements (note that this is a different notion than that of ccc in Boolean algebras). A subset of $\boldsymbol{P}$ is a filter if it is directed and downward closed as a partial order. A subset $D$ of $\boldsymbol{P}$ is dense if for every $p \in \boldsymbol{P}$ there is $q \in D$ with $p \leq q$. MA states that for any ccc forcing $\boldsymbol{P}$ and any family $\mathcal{F}$ of $<2^{\mathrm{\aleph}_{0}}$ dense subsets of $\boldsymbol{P}$, there is a filter of $\boldsymbol{P}$ which intersects each of the dense sets in the family. In applications, one may start with a goal of constructing some object, usually of size $\boldsymbol{\kappa}_{1}<2^{\boldsymbol{\aleph}_{0}}$ (which is why the negation of $\mathrm{CH}$ is usually assumed as well), and $\boldsymbol{P}$ consisting of (usually) finite pieces of the desired object, ordered so that a stronger element of $\boldsymbol{P}$ gives more information about the object than a weaker one. Then one needs to formulate a family $\mathcal{F}$ of dense sets which represent various requirements on the object constructed, and the $\mathcal{F}$-generic filter guaranteed to exist by MA will, in some natural way, give rise to the object desired.

In the context of theorem 4.3, in the non-trivial direction from (i) to (ii) and in view of the characterization from theorem 4.1, it would be natural to start with a Boolean algebra, $\mathfrak{B}$, which satisfies ccc and has size $<2^{\mathfrak{x}_{0}}$, and to formulate a ccc forcing notion, which would force a non-atomic strictly positive measure on $\mathfrak{B}$. One would need to formulate a dense set corresponding to the requirement that $a$ has positive measure, for every non-zero $a$ in $\mathfrak{B}$. A posteriori this may be proven possible, but this is not how our proof went. In addition, the known proof that every ccc Boolean algebra of size $<2^{\mathbf{x}_{0}}$ is separable and hence satisfies Kelley's condition

\footnotetext{
${ }^{1}$ Some authors use partially ordered sets with the largest element, in which case notions we define
} need to be read with $\geq$ in place of $\leq$. The present notation follows Cohen (1963). 
and carries a measure (see Fremlin 1984), is not useful since it gives an atomic measure. We worked instead with the notion of approximability. A Boolean algebra $\mathfrak{B}$ is approximable if there is a sequence $\left\langle\mu_{n}: n<\omega\right\rangle$ of probability measures on $\mathfrak{B}$ such that for every $a \neq 0_{\mathfrak{B}}$ in $\mathfrak{B}$ there is $n$ such that $\mu_{n}(a)>1 / 2$. This notion was studied by Talagrand (1980) and Mägerl \& Namioka (1980) with the idea of characterizing Boolean algebras that support a strictly positive separable measure (SM). We shall discuss this in a moment, but for now let us finish sketching the proof of theorem 4.3. The proof is to start with a Boolean algebra that satisfies Kelley's condition (in fact a weaker condition than that) and formulate a ccc forcing notion whose generic filter gives a sequence $\left\langle\mu_{n}: n<\omega\right\rangle$ which demonstrates that the algebra is approximable. The size of the family $\mathcal{F}$ of sets such that we need to produce an $\mathcal{F}$-generic filter is equal to the size of $\mathfrak{B}$, so if MA holds, we can deal with any ccc algebra of size $<2^{\aleph_{0}}$. Having the sequence $\left\langle\mu_{n}: n<\omega\right\rangle$, we can define the weighted $\operatorname{sum} \sum_{n<\omega} \mu_{n} / 2^{n+1}$, and the proof is done in such a way that if the algebra is atomless then this measure in non-atomic.

Having sketched the proof of theorem 4.3, we can discuss the next item on our list, namely the notion of SM defined earlier. Talagrand (1980) attacks MRP (separable) by defining a list of properties of decreasing strength one of which is 'supporting a strictly positive SM'. He shows that the property of $\mathfrak{B}$ being $\sigma$-centred (so the algebra can be written as a countable union of subfamilies each of which has the property that for all finite subsets $J$, we have $\cap J \neq \emptyset$, provided $\emptyset \notin J)$ is strictly stronger than SM, while SM implies approximability. He also shows that under the assumption of $\mathrm{CH}$, property $\mathrm{SM}$ is strictly stronger than approximability. There is a possibility left open by this theorem, which is that approximability might, under some suitable axioms, actually characterize SM. Džamonja \& Plebanek (submitted) show that this is not the case:

Theorem 4.4. There is a Boolean algebra which is approximable but does not support a strictly positive separable measure.

Proof of this theorem involves a combinatorial construction, the details of which are out of the scope of this paper. A major building block is a zerodimensional topological space without isolated points with the curios property that it has a countable dense set $D$, such that for every sequence $\left\langle F_{n}: n<\omega\right\rangle$ of closed sets whose union is disjoint from $D$, that union is nowhere dense. Such a space was first constructed in ZFC by Simon (2002), although it was known before that such spaces can exist under various additional axioms of set theory.

Similar to Talagrand (submitted), by answering negatively the problem of von Neumann brings us back to square one regarding MRP (countably additive), so does theorem 4.4 brings us back to the beginning regarding MRP (separable). It would have been very nice if at least consistently there would be an equivalence between approximability and separability, since there is a combinatorial characterization of approximability due to Mägerl \& Namioka (1980):

Theorem 4.5 (Mägerl-Namioka). A Boolean algebra $\mathfrak{B}$ is approximable if and only if for every $\varepsilon>0$ (equivalently: for some $\varepsilon \in(0,1)$ ) there is a decomposition $\mathfrak{B} \backslash\{0\}=\cup_{n<\omega} \mathfrak{B}_{n}^{\varepsilon}$, where for each $n$ we have $\operatorname{int}\left(\mathfrak{B}_{n}^{\varepsilon}\right) \geq 1-\varepsilon$.

In the absence of such an equivalence, we have to rethink what a possible characterization may look like. At this point, we may say that it is unlikely that any characterization will involve only a decomposition into countably many pieces 
each of which has some given property, in the style of all but one characterization given here. Instead, the desired theorem will probably have to take into account the interaction between these various pieces, like in theorem 4.1. The reason for this is a result by Dow \& Steprans (1993). Namely, one of the strongest possible characterizations involving only the information about each of the countably many pieces (given that we know that $\sigma$-centredness is too strong) would use the notion of $\sigma$-n-linkedness. For a given $n$, a family $A$ of non-empty sets is $n$-linked if for any subset $J$ of $A$ of size $\leq n$, we have $\cap \neq \emptyset$. A Boolean algebra is $\sigma$-n-linked if it can be written as a countable union of sets each of which is either $\{\emptyset\}$ or $n$-linked. Dow \& Steprans (1993) proved that the measure algebra of $[0,1]^{\kappa}$ is $n$-linked for every $n$ if and only if $\kappa \leq 2^{\mathrm{\aleph}_{0}}$. This shows that the notion of $n$-linkedness does not make a sufficient distinction between SM algebras and those of type $\leq 2^{\mathbf{x}_{0}}$.

\section{Conclusion}

This paper shows on the specific example of the MRP the phenomenon of the utility of a multidisciplinary mathematical approach to a single mathematical problem, in particular, the value of a set-theoretic analysis of the problem at hand. We have shown how MRP, which is a problem about Boolean algebras that was asked first at least in 1937 if not earlier, has had an impact on measure theory, combinatorics and set theory, and in turn, that each of these subjects contributed to a better understanding of MRP. Specifically, we discussed the set-theoretic insights and emphasized the point of using set-theoretic tools and being able to obtain a purely measure-theoretic or algebraic final result. Through historical remarks presented in the first parts of the paper, the reader can see how various results have followed the current state of knowledge, both in set theory and measure theory. In $\S 4$, we showed some new results and underlined the future directions of research in the subject. One of the goals is a structure theory for Boolean algebras that support a finitely additive strictly positive measure.

\section{Notation}

The power set of a given set $A$ is the set of all subsets of $A$ and is denoted by $\mathcal{P}(A)$.

The set-theoretic notation $\left\{E_{n}: n<\omega\right\}$ is used for what some authors denote by $\left\{E_{n}: n \in \boldsymbol{N}\right\}$ or (after a re-enumeration) $\left\{E_{n}: 1 \leq n<\infty\right\}$. Our notation for sequences is $\left\langle a_{n}: n<\omega\right\rangle$ in place of $\left(a_{n}\right)_{n}$. A similar convention is used for $\sum_{n<\omega}$.

Many notions were introduced in the text, where at first appearance they were italicized.

The author thanks EPSRC for their support through an Advanced Fellowship in Mathematics and the British Council for support through an Alliance Grant for years 2005 and 2006.

\section{References}

Balcar, B., Jech, T. \& Pazák, T. 2005 Complete ccc boolean algebras, the order sequential topology, and a problem of von Neumann. Bull. Lond. Math. Soc. 137, 885-898. (doi:10.1112/ S0024609305004807) 
Bhaskara Rao, K. P. S. \& Bhaskara Rao, M. 1983 Theory of charges. New York, NY: Academic Press.

Cantor, G. 1874 Über eine Eigenschaft des Inbergriffs aller reellen algebraischen Zahlen. J. f. Math. 77, 258-262.

Cohen, P. 1963 The independence of the continuum hypothesis. Proc. Natl Acad. Sci USA 50, 1143-1148. (doi:10.1073/pnas.50.6.1143)

Dow, A. \& Steprans, J. 1993 The $\sigma$-linkedness of measure algebra. Can. Math. Bull. 37, 42-45.

Džamonja, M. \& Plebanek, G. Submitted. Measures on Boolean algebras.

Fremlin, D. H. 1984 Consequences of Martin's axiom. Cambridge, UK: Cambridge University Press.

Fremlin, D. H. 1989 Measure algebras. In Handbook of Boolean algebras III (ed. J. D. Monk), pp. 877-980. Amsterdam, The Netherlands: North-Holland.

Fremlin, D. H. 2002 Measure theory (measure algebras), vol. 3. Colchester, CT: Torres Fremlin.

Gaifman, H. 1964 Concerning measures on Boolean algebras. Pacific J. Math. 14, 61-73.

Gödel, K. 1931 Über formal unentscheidbare Sätze der Principia Mathematica und verwandter Systeme I. Monatsh. Math. Phys. 38, 173-198. (doi:10.1007/BF01700692)

Kelley, J. L. 1959 Measures on Boolean algebras. Pacific J. Math. 9, 1161-1177.

Mägerl, G. \& Namioka, I. 1980 Intersection numbers and weak* separability of spaces of measures. Math. Ann. 249, 273-279. (doi:10.1007/BF01363900)

Maharam, D. 1942 On homogeneous measure algebras. Proc. Natl Acad. Sci. USA 28, 108-111. (doi:10.1073/pnas.28.3.108)

Maharam, D. 1947 An algebraic characterization of measure algebras. Ann. Math. 48, 154-167. (doi:10.2307/1969222)

Maharam, D. 1981 Commentary on problem 163. In The Scottish book (ed. R. D. Mauldin), pp. 241-243. Boston, MA: Birkhaüser.

Shelah, S. 1994 Cardinal arithmetic. Oxford, UK: Oxford University Press.

Simon, P. 2002 A countable dense-in-itself dense P-set. Topol. Appl. 123, 193-198. (doi:10.1016/ S0166-8641(01)00182-1)

Stone, M. H. 1936 The theory of representations for Boolean algebras. Trans. Am. Math. Soc. 40, 37-111. (doi:10.2307/1989664)

Talagrand, M. Submitted. Maharam's Problem.

Talagrand, M. 1980 Séparabilité vague dans l'espace des mesures sur un compact. Israel J. Math. 37, 171-180.

Todorčević, S. 2004 A problem of von Neumann and Maharam about algebras supporting continuous submeasures. Fundam. Math. 183, 169-183.

Veličković, B. 2005 CCC forcings and splitting reals. Israel J. Math. 147, 209-220.

von Neumann, J. 1981 Problem 163. In The Scottish book (ed. R. D. Mauldin), p. 240. Boston, MA: Birkhaüser. 


\title{
AUTHOR PROFILE
}

\author{
Mirna Džamonja
}

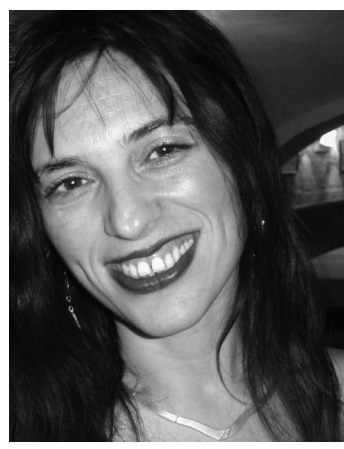

Mirna Džamonja was born in Sarajevo, former Yugoslavia and now Bosnia and Herzegovina. She studied at the University of Sarajevo where she graduated as the best student of the year in mathematics in 1988. She then moved to the University of Wisconsin-Madison, USA and obtained her M.A. in 1990 and Ph.D. in 1993, both in mathematics. She was a postdoc and a Lady Davis Scholar at the Hebrew University of Jerusalem in Israel until 1995, when she obtained a Van Vleck Visiting Assistant Professorship at the University of Wisconsin-Madison. In 1998 she moved to Britain, where she took a lectureship at the university of East Anglia in Norwich. Since 2002 she has been a reader there. In 2001 she was awarded an EPSRC Advanced Fellowship which she still holds. Her visiting positions include a Logic Professorship at UW-Madison, Guest Professorship at the University of Paris VII, Associate Professorship at CU New York and Senior Researchership at the University of Vienna. Mirna's main area of research is set theory, but she has broad interests in mathematics, and is an author of papers in topology, model theory and measure theory. She has written about 40 papers and is writing a book on the universality problem. She is a member of the editorial board of the Sarajevo Journal of Mathematics, a review editor of the Bulletin of Symbolic Logic and the secretary to the British Logic Colloquium. 